\title{
Research on the Segmentation and Pick-up Algorithm of Bamboo Wood Cross-section Based on Lab Color Space
}

\author{
WANG Hai-feng ${ }^{1,2}$ and REN Hong-e ${ }^{1 *}$ \\ ${ }^{1,}$ Information and Computer Engineering College, Northeast Forestry University, \\ Harbin, 150040, P.R. China; \\ ${ }^{2}$ School of Electronic and Information Engineering, Qiongzhou University, \\ Sanya, Hainan, 572022, P.R. China \\ renhonge163@163.com;wyfxxz@163.com
}

\begin{abstract}
The accurate segmentation and extraction of bamboo cross-section image has a vital role on bamboo processing automation. The Lab color space in accordance with the color features of the bamboo wood cross-section is chosen in this paper. The bamboo crosssection image segmentation and extraction algorithm based on the clustering theory is proposed. The algorithm firstly takes advantage of the character that the colors represented by channel $a$ and channel $b$ of the Lab color space accord with the color of the bamboo wood, to be combined feature vector. Then the algorithm uses the k-mean clustering algorithm to classify the eigenvectors to realize the segmentation of the bamboo wood cross-section. At last the circle fitting algorithm is used to realize the final frame of the bamboo wood cross-section. The results of the experiments show that the algorithm can be used to realize the complete segmentation of the cross-section image of bamboo wood, and to frame the results correctly, the time performance of which can meet the requirements of the subsequent processing.
\end{abstract}

Keywords: Image segmentation, Target extraction, Lab color space, Bamboo wood cross-section

\section{Introduction}

With the fast economic development of China, there is an increasing demand on wood in economic construction and human life. At present, the forestry in our country is experiencing a historical change from dominated by lumber production to mainly for the ecological construction, especially with the implementation of natural forest resources protection project, which results in the sharp decline in the timber output and an obvious increase in import. As China has the most abundant bamboo resources, which is one of the countries with the largest areas of bamboo forests and the most production, as a new material with the characteristics of low carbon, environment friendliness, energy conservation and emission reduction, ecology, innovation, sustainable development and national features, bamboo material has become a good substitute of wood and will be a mainstream material in the future. The traditional bamboo processing technology uses the transverse circular saw to cut bamboo wood into segments, and then uses the sphenoid separator of the mechanical bamboo breaking machine to cut the circular bamboo wood segments apart. Only by manually changing the separators with different numbers of blades according to the diameters of the bamboo wood, the radians of the bamboo pieces can be guaranteed to be equal. Besides, to enhance machining precision, in the operation, the geometric center of the two ends of the bamboo piece needs to be in alignment with the center of the separator and the conical tip of the stop plate cone [1]. The manual loading of material and changing of blades in operation waste time and labor, which makes it hard to realize automatic processing. However, it is significant for the using 
efficiency of bamboo wood to process the bamboo wood fast and effectively. In order to improve the processing technology, efficiency and the evaluation of the processing effects in processing, the image recognition based technology is adopted in bamboo wood processing, and the numerical control and automation technologies are introduced into the field of bamboo wood processing. The recognition of the bamboo wood image functions vitally in bamboo wood processing, and though there are a lot of target recognition methods based on image $[2,3,4]$, these methods are not in common use due to target differences. Besides, because the situations on the work site are complex, the partition method on gray-scale map is easy to be disturbed by the background, and greatly influenced by weather and light, while color images provide richer information than gray level images. The partition of color images not only needs to choose proper color image, but also to adopt partitioning algorithm fit for this space. Depending on bamboo wood and its color image features, this paper uses imaging sensors to collect the cross-section image data of bamboo wood, to analyze and process, then presents the color image partition method based on Lab color space, which utilizes the repeatability of bamboo wood color ranges of the spaces $a$ and $b$ in the Lab color image, mixes the color spaces of $\mathrm{a}$ and $\mathrm{b}$ to compose feature vector, adopts kmean clustering partition, through filtering to get the cross-section image of the bamboo wood, and finally setting the frame of the bamboo wood cross-section using circular fitting algorithm. The algorithm can fast, accurately and effectively recognize the cross-section of the bamboo wood, judge the quality and effect of the bamboo wood processing technology according to the bamboo wood image, and instruct the next step processing, which has a significant meaning in realizing the automatic processing of bamboo wood.

\section{Color Space and Transformation}

Color space, also known as color model, has a wide range, with the color spaces RGB, $\mathrm{CMY}$, Lab etc commonly used at present. In the partition of color images, the selection of color spaces is the most important. Because most of the color spaces are part change or exclusively used in some field, the selection of color space will directly influence the methods adopted in partition and the final results of the partition. RGB is the most commonly used color space, which regards colors to be the combination of three primary colors (red, green, blue), the different ratios of which will produce different colors. And there are a lot of applications on RGB color space, such as document [5] using RGB color space and textual features to compose feature vector and realize the surface grading of bamboo segment. Document [6] extract the color features of license plates in RGB color space, to design a method to check license plates and to realize the location of license plates. However, the three components of RGB have strong correlation, a lot of redundant information and a large amount of computation [7], and the models can only reflect the changes of the color gradients of the pixel points in the images, while cannot show the differences among hues, so it is not fit to be used directly in the image segmentation through independent computation on the basis of the three components.

In 1976, Commission Internationale de l'éclairage (CIE) formulated a kind of color mode - CIE Lab color space, regarded as the approximate of the uniform color space by CIE [8], which has three basic coordinates $\mathrm{L}, \mathrm{a}, \mathrm{b}$, with $\mathrm{L}$ representing brightness, $\mathrm{L}=0$ generating black, while $\mathrm{L}=100$ indicating white; $\mathrm{a}, \mathrm{b}$ are the color passageways, with a value range from +127 to -128 , a of a positive number representing red, of a negative number representing green; $b$ of a positive number representing yellow, of a negative number representing blue. The sketch map of Lab color space is shown in Figure 1. Through which it can be seen that the Lab model is a 3D model, which can only be fully shown in a three dimensional space. Lab colors are designed to approximate human vision, focusing on sensing uniformity, and the component $\mathrm{L}$ bears a close relationship with human brightness perception. Lab space is a display mode used to describe colors, while not the number of specific pigments needed in the color generation of equipment, 
so Lab is regarded as a color model irrelevant to equipment [9]. The color gamut of the Lab space is larger than other color spaces, which nearly contains all the colors human can perceive, and many "colors" in Lab space are beyond the human sight, so they are purely hypothetical and cannot be reproduced in the physical world.

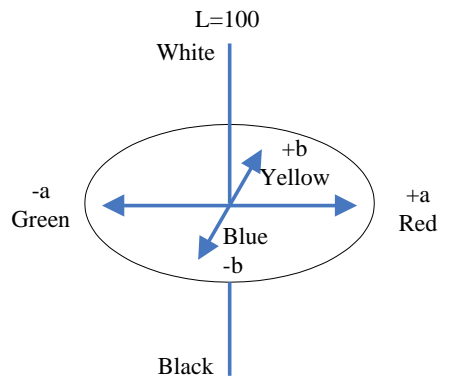

Figure 1. Lab Color Space

CIE Lab is directly based on CIE XYZ color space, and it needs to rely on XYZ to transform RGB color space into Lab color space, so first it needs to transform RGB color space into XYZ space, as formula (1) shows, then to transform XYZ space into Lab color space shown as formula (2) [3].

$$
\begin{aligned}
& {\left[\begin{array}{l}
X \\
Y \\
Z
\end{array}\right]=}\left(\begin{array}{lll}
0.412453 & 0.357580 & 0.180423 \\
0.212671 & 0.715160 & 0.072169 \\
0.019334 & 0.119193 & 0.950227
\end{array}\right)\left[\begin{array}{l}
R \\
G \\
B
\end{array}\right] \\
& L^{*}=116 \times f\left(\frac{Y}{Y_{n}}\right)-16 \\
& a^{*}=500 \times\left[f\left(\frac{X}{X_{n}}\right)-f\left(\frac{Y}{Y_{n}}\right)\right] \\
& \text { In which, } f(s)=\left\{\begin{array}{c}
b^{*}=200 \times\left[f\left(\frac{Y}{Y_{n}}\right)-f\left(\frac{Z}{Z_{n}}\right)\right] \\
\frac{1}{3}\left(\frac{29}{6}\right)^{2} s+\left(\frac{4}{29}\right) \quad s \leq\left(\frac{6}{29}\right)^{3}, \text { here } X_{n}, Y_{n}, Z_{n} \text { are the }
\end{array}\right.
\end{aligned}
$$
reference white points of the tristimulus values, the values of which are 0.0504, 1.0000, 1.0887 .

\section{Research on the Bamboo Image Segmentation Algorithm}

\subsection{Feature Extraction based on Lab Color Space}

The color images contain richer image information, and have many kinds of color space presentations, so the key of the color image segmentation lies in how to utilize the rich color information to achieve the purpose of segmentation [10]. The color of timber is determined by the lignin in it, so similarly the amount of lignin in bamboo wood will also determine the generation and changes of colors in its surface. Hou yanling [11], through experiments, found that the surface brightness indexes of bamboo wood did not change significantly along with the increase of the ages of bamboo wood of different ages, and 
the surface colors of bamboo wood of different ages did not change greatly along with age increase. In its Lab color space, the value of passageway a is around 0, and the value of passageway $b$ is around 30, which can provides basis for the use of Lab color space to segment bamboo images. Figure 2 is the color space figure of the experimental bamboo wood in Lab space.

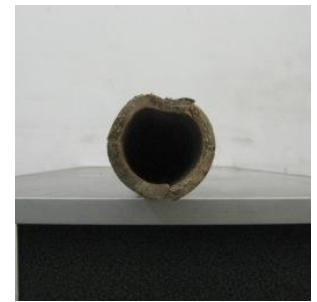

(a) original image

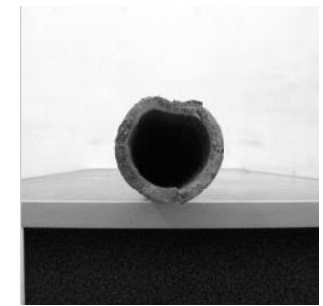

(b) $\mathbf{L}$

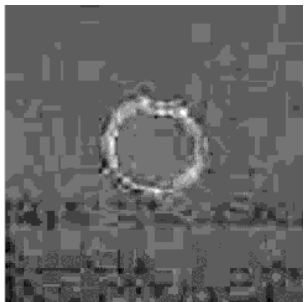

(c) $\mathbf{a}$

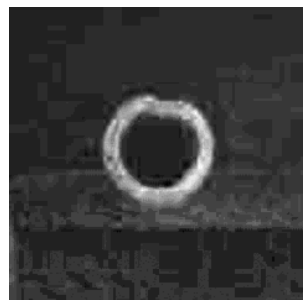

(d) b

Figure 2. Lab Color Space of the Bamboo Wood Image

Because $\mathrm{L}$ is lightness, relevant to human perception, it is not taken into consideration here, and through experimental measurement we get: a, b spaces will both influence the segmentation results. And because the system requires a high level of real-time performance, it must adopt the identification methods which needs a small amount of computation but the speed of which is fast, so a, b spaces are chosen to make hybrid feature vector, details of which are shown in formula (3)

$$
f e a(m, n)=(1-\lambda) * a(m, n)+\lambda * b(m, n)
$$

Here $\lambda$ is an adjustable parameter, set as 0.2 here.

The Figure image formation of the original image is shown in formula (4).

$$
\text { Feature_Image }(m, n)=\{f e a(m, n), a(m, n)\}
$$

The noise in the image will affect the segmentation results, so noise reduction needs to be done for the image, where Gauss Low-pass Filtering $h^{\prime}(x, y)=\frac{1}{2 \pi \sqrt{\sigma}} e^{-\frac{\left(x^{2}+y^{2}\right)}{2 \sigma^{2}}}$ is adopted to do noise reduction for passageway a and passageway b.

\subsection{Bamboo Wood Cross-section Image Segmentation and Circle Fitting Frame Algorithm}

The specific procedures of the image segmentation algorithm of bamboo wood crosssection based on Lab color space are as follows:

(1) Switch the collected RGB images to Lab color space.

(2)Extract the color information of passageways a and $b$, to compose feature vectors according to formula (4).

(3)Use kmean clustering to classify the feature vectors.

(4)Adopt morphological operation to remove small areas, acquire the cross-section image of the segmented bamboo wood.

Because the bamboo wood cross-section image may slant, this will result in that the bamboo wood cross-section cannot be acquired directly through the segmented image, so here it needs to frame the cross-section of the bamboo wood. The cross-section is round, so the circle fitting method is used to carry out the framing. There are more than one circle fitting algorithms, here the three-point fitting circle method in [12] is taken as a reference. Suppose A, B, C are the three points on the extracted bamboo wood crosssection image, shown in Figure 3. The coordinates of them are respectively $A\left(x_{a}, y_{a}\right)$, $B\left(x_{b}, y_{b}\right), C\left(x_{c}, y_{c}\right)$, and angle $\mathrm{CAB}$ is $\mathrm{r}$. Draw a vertical $\mathrm{OM}$ from the circle center $\mathrm{O}$ 
to line $\mathrm{BC}$ to get $\mathrm{M}$ as the midpoint of $\mathrm{BC}$, and the coordinate of $\mathrm{O}$ as well as the radius $\mathrm{R}$ can be derived and calculated by formula (5) and (6).

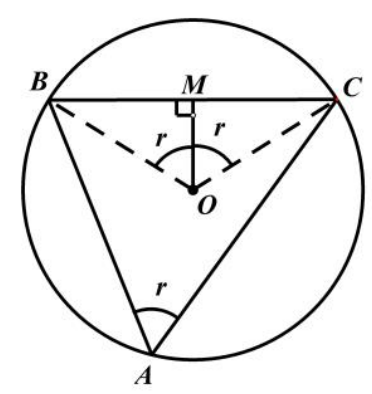

Figure 3. Three Points Fitting Circle Sketch Map

$$
\begin{aligned}
& O_{x}=\frac{1}{2}\left[x_{b}+x_{c}+\left(y_{b}-y_{c}\right) \cdot c \tan r\right] \\
& O_{y}=\frac{1}{2}\left[y_{b}+y_{c}+\left(x_{c}-x_{b}\right) \cdot c \tan r\right] \\
& R=S_{B C} / 2 \sin r
\end{aligned}
$$

In which, $S_{B C}$ is the length of segment BC.

The specific framing algorithms are as follows:

(1)As for the result image after segmentation, remove the small areas and acquire the biggest part of segmented image.

(2)Get the four boundary points left, right, top, bottom of the segmented bamboo wood cross-section image.

(3)Suppose the four boundary points are the bamboo wood end face (assumed to be perfect circle) to be segmented, so the intersection A of the straight line formed by the two points of top and bottom and the straight line formed by points left and right will be the center of the circle.

(4)Make a circle fitting respectively for every three of the four points above, get the center of the circle and the radius by using formula $(5,6)$ respectively, and choose the point which is nearest to point $\mathrm{A}$ from the four circle centers after fitting to be the center of the circle finally framed, to get the outer boundary of the bamboo section.

\section{The Experiment and Result Analysis}

In order to testify the accuracy and effectiveness of the algorithms raised, a segmentation experiment is done on a bamboo wood section image sized $256 \times 256$. The algorithm experiment is performed under the environment of Windows 7, and the testing environment is a notebook computer with $2.4 \mathrm{GHZ} \mathrm{CPU,} 3 \mathrm{~GB}$ memory storage, all of the algorithms is realized under MATLAB 7.0. Figure 4 is the Original images, segmentation and framing results of the four bamboo wood cross-sections collected.
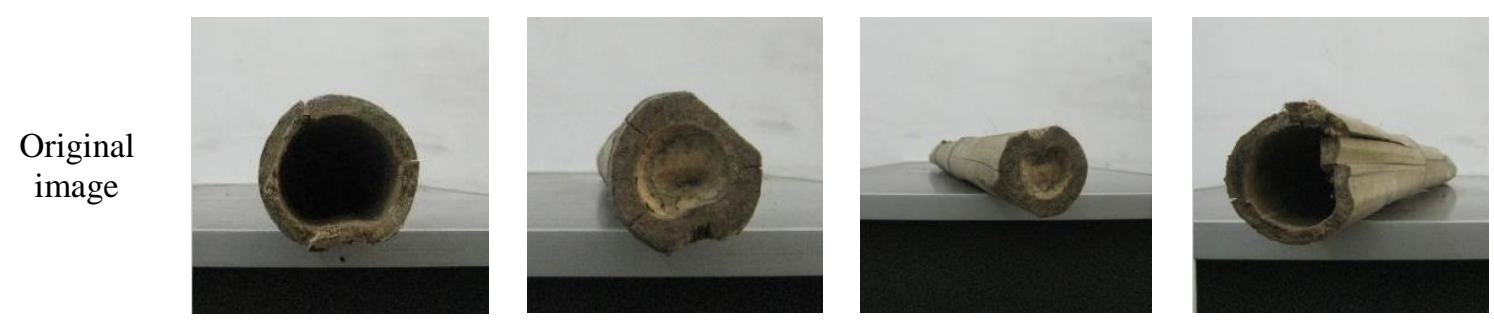


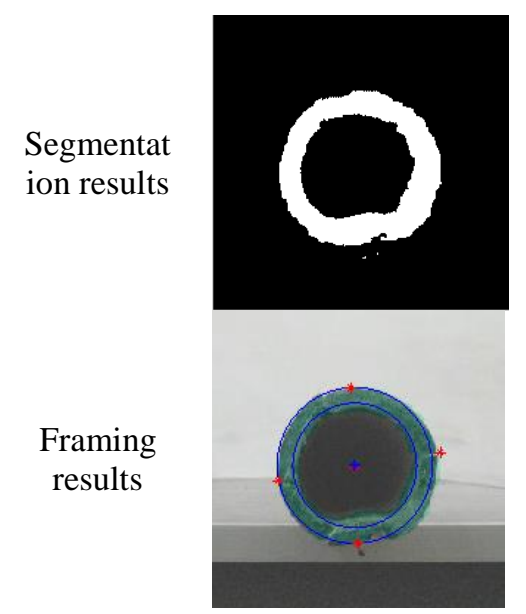

(a)

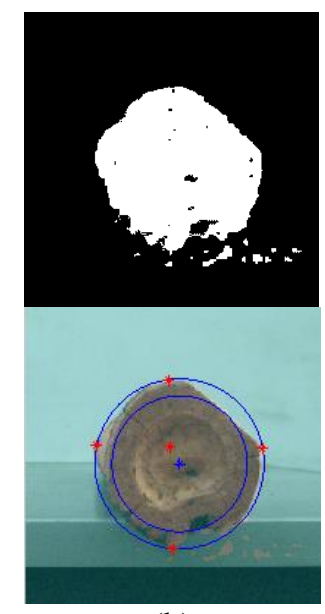

(b)

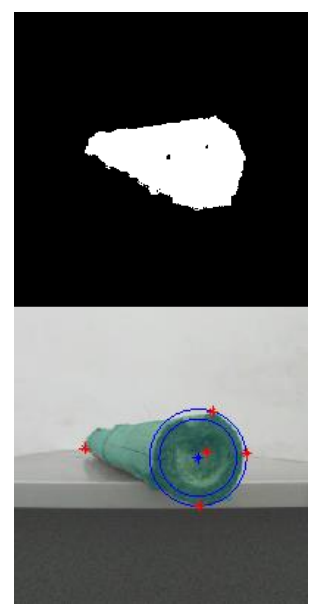

(c)

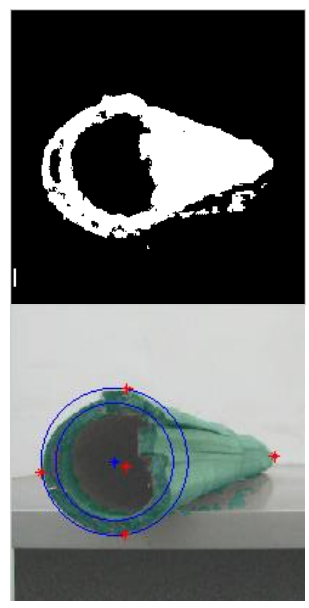

(d)

Figure 4. Segmentation and Framing Results

In Figure 4, the first line is the original images to be segmented, and the second line is the segmentation results acquired after adopting the image segmentation method of 3.2, which shows that the method presented in this paper can successfully separate full bamboo from the background, with a good segmentation result, and a high robustness of the algorithm; The third line in the Figure is the framing results got after adopting the circle fitting algorithm in 3.2 , with the blue $*$ representing the circle centers acquired in the end, and red points as the four boundary points and their intersection A. From the experimental results of framing, it can be seen that as for the frontal bamboo wood image (image a), the segmentation and framing of its section can be done accurately, and as for images (images c, d) slanting to the right or the left, it can also accurately frame the sections of the bamboo wood, and the framing of image $b$ is wrong because the section of bamboo wood image is not regular and has a small slant angle and the four points are not on the same circle.

To testify the real time performance of the algorithm, try to stop other application programs during the experiment, all of the algorithms are implemented for 5 times, the average time consumed of which is used as the run time of this algorithm, with the specific results show in Table 1. It can be seen that the algorithm of this paper can meet the requirement of real-time processing.

Quantitative evaluation is done on the segmentation results, using formula (7) to calculate segmentation accuracy.

$$
\text { SegmentationAccuracy }=\begin{aligned}
& \text { the number of the same pixels in the results } \\
& \text { of segmentation and manual segmentation }
\end{aligned} / \mathrm{M} \times \mathrm{N}
$$

In which, $\mathrm{M}, \mathrm{N}$ are the pixels of the image. Here the manual segmentation result is the image got after segmenting the full bamboo by hand, the size of which is the same as that of the source image. The segmentation accuracy is shown in Table 1.

\section{Table 1. The Run Time of the Algorithm and the Accuracy of the Image Segmentations}

\begin{tabular}{c|c|c}
\hline Image No. & Run time (second) & Segmentation accuracy \\
\hline (a) & 0.9762 & $98.95 \%$ \\
(b) & 0.8940 & $98.19 \%$ \\
(c) & 0.8674 & $99.29 \%$ \\
(d) & 0.8596 & $97.26 \%$ \\
\hline
\end{tabular}


In order to testify the accuracy of framing algorithm, manually use the software of Photoshop to calculate the pixel number of the diameter of the bamboo wood section, and compare it with the pixel number of the diameter identified by the presented algorithm, the data of which are shown in Table 2. The maximum deviation is within $\pm 10 \%$, which can meet the accuracy requirements of the system.

\section{Table 2. The Computation Results and Deviations of the Algorithm Presented in this Paper}

\begin{tabular}{c|c|c|c}
\hline Image No. & $\begin{array}{c}\text { Manually } \\
\text { calculated cross- } \\
\text { section diameter } \\
\text { pixel number }\end{array}$ & $\begin{array}{c}\text { Presented algorithm } \\
\text { identified diameter } \\
\text { pixel number }\end{array}$ & $\begin{array}{c}\text { Deviation } \\
\text { percentage }\end{array}$ \\
\hline (a) & 135 & 134 & $0.75 \%$ \\
(b) & 137 & 146 & $-6.16 \%$ \\
(c) & 83 & 84 & $-1.19 \%$ \\
(d) & 123 & 120 & $2.5 \%$ \\
\hline
\end{tabular}

From the performance time, segmentation accuracy and the deviation of calculated results of the algorithm, the system can meet the requirement of real-time online testing. After accurately identifying the bamboo wood image and measuring the cross-section diameter of the bamboo wood, the computer will send an output result to the bamboo wood manufacturing system which judges the next mechanical action and gets ready for it.

\section{Conclusion}

The paper adopts the clustering image segmentation method based on Lab color space, to realize automatic recognition, segmentation and framing. The algorithm is not influenced by illumination variation, which can acquire a good result of segmentation and accurate framing. Moreover, the computational process of the algorithm is simple with a high level of real-time performance, which can meet the needs of bamboo wood machinery automatic processing. It has a very good application prospect to apply CIE Lab color space most widely used currently to identifying bamboo wood cross-section, though because of the complex situations in the work places, there are still some problems in the algorithm presented in this paper, such as over-segmentation or not enough segmentation, resulting in incorrect final segmentation results, leading to mistakes in the next step of processing, all of which need to be further improved and studied in future work.

\section{ACKNOWLEDGEMENTS}

The work was supported by Chinese National Forestry Public Program (201204715), Key Projects for natural science foundation of Heilongjiang province (ZD201203/C1603), the National Natural Science Foundation (31370566), Scientific Research Foundation of Qiongzhou University (No. QYQN201326), The key scientific and technological plan projects of Hainan (No.zdxm2014087), The scientific and technological cooperative project for college and region of Sanya (2014YD14, 2014YD30, 2014YD24).

\section{References}

[1] L. Baiping, "Manufacturing Technology of Bamboo Panels Made from Laminated Circumferential Sections", Northeast Forestry University, (2010).

[2] K. Hasung and H. Song, "Study on the Feature Extraction of Face using Color Space", 2012 International Conference on Advances Science and Contemporary Engineering (ICASCE 2012), Procedia Engineering, vol. 50, (2012), pp. 668-674. 
[3] J. Hernandez, A. Quintanilla, J. Lopez, et al., "Detecting objects using color and depth segmentation with Kinect sensor", Procedia Technology, vol. 3, (2012), pp. 196-204.

[4] D. Jiajia, "The Research of Power Battery Welding Point Positioning Technology Based on the Color Space", North University of China, (2012).

[5] X. Wang, D. Lianga and W. Deng, "Surface grading of bamboo strips using multi-scale color texture features in eigenspace", Computers and Electronics in Agriculture, vol. 73, no. 1, (2010), pp. 91-98.

[6] Z. Chengyong, "A novel license plate location method on RGB color space", Journal of Image and Graphics, vol. 11, no. 15, (2010), pp. 1623-1628.

[7] W. Xiali, Z. Mingquan and G. Guohua, "An Approach of Vehicle Plate Extract Based on HSV Color Space", Computer Engineering, vol. 17, no. 30, (2004), pp. 133-135.

[8] A. Jain, "Fundamentals of digital image processing", New York: Prentice Hall, (1989).

[9] S. He, Z. Liu and L. Wang, "Color Calibration and Color Management in Digital Camera", Future Communication, Computing, Control and Management. Springer Berlin Heidelberg, (2012), pp. 579585.

[10] I.-U.-H. Qazi, O. Alata, J.-C. Burie, A. Moussa and C. Fernandez-Maloigne, "Choice of a pertinent color space for color texture characterization using parametric spectral analysis", Pattern Recognition, vol. 44, no. 1, (2011), pp. 16-31.

[11] H. Yanling, "Study on Surface Wettability and Color of Moso Bamboo (Phyllostachys Pubescens)", Inner Mongolia Agricultural University, (2010).

[12] Z. Yu and J. Lin-Jia, "Synthesis Tracing Algorithm for Region Localization of SEM Image", Acta Photonica Sinica, vol. 2, no. 37, (2008), pp. 391-394.

\begin{abstract}
Authors
Wang Haifeng, he was born in 1980 . He received the M.S. degree in college of information and computer Engineering from northeast forestry university in 2009. He is a lecturer with College of Electronics and Information Engineering, Qiongzhou University, Sanya,PR China. Since 2012 he has been pursuing his $\mathrm{PhD}$ degree in northeast forestry university. His research interests include computer vision, image segmentation, pattern recognition, forestry intelligent equipment and Information control forestry.

Honge REN, she was born in 1962. She received the Ph.D. degree from Northeast Forestry University, China, in 2009. She is currently professor of information and computer engineering college at school of Northeast Forestry University, supervisor of Dr. Her main research interests include the pattern recognition and intelligent control.
\end{abstract}

